\title{
Different moment-angle manifolds arising from two polytopes having the same bigraded Betti numbers
}

\author{
SuYOUNG CHOI
}

\begin{abstract}
Two simple polytopes of dimension 3 having identical bigraded Betti numbers but nonisomorphic Tor-algebras are presented. These polytopes provide two homotopically different moment-angle manifolds having the same bigraded Betti numbers. These two simple polytopes are the first examples of polytopes that are (toric) cohomologically rigid but not combinatorially rigid.
\end{abstract}

55N99; 05A15

\section{Introduction}

A convex polytope of dimension $n$ is called simple if there are exactly $n$ facets (codimension one face) meeting at each vertex. Let $P$ be an $n$-dimensional simple convex polytope with $m$ facets $F_{1}, \ldots, F_{m}$. Consider an $m$-dimensional real compact torus $T^{m}$, and denote the $i^{\text {th }}$ coordinate subgroup of $T^{m}$ by $T_{i}$.

Definition 1.1 Consider the following equivalence relation on $T^{m} \times P$ :

$$
(t, p) \sim\left(t^{\prime}, p^{\prime}\right) \Longleftrightarrow p=p^{\prime}, t^{\prime} t^{-1} \in \bigoplus_{F_{i} \ni p} T_{i} .
$$

The quotient space

$$
\mathcal{Z}_{P}=\left(T^{m} \times P\right) / \sim
$$

is called the moment-angle manifold of $P$ and is denoted by $\mathcal{Z}_{P}$.

Observe that $\mathcal{Z}_{P}$ is indeed a manifold of dimension $m+n$ (see Buchstaber and Panov [2, Lemma 6.2]), and the formula $s \cdot[t, p]=[s t, p]$ defines a natural $T^{m}$-action on $\mathcal{Z}_{P}$ with orbit space $P$. The moment-angle manifold was first introduced in Davis and Januszkiewicz [7] as a space that has the following universal property: for every quasitoric manifold (the definition will be given below) $\pi: M \rightarrow P$, there is a principal $T^{m-n}$-bundle $\mathcal{Z}_{P} \rightarrow M$ whose composite with $\pi$ is the orbit map $\mathcal{Z}_{P} \rightarrow P$. Hence, it is one of the key concepts in toric topology, so it is very important to study the topology of $\mathcal{Z}_{P}$. 
A formula for the cohomology of $\mathcal{Z}_{P}$ has already been established. Let $\mathbb{k}$ be a field, and let $A=\mathbb{k}\left[v_{1}, \ldots, v_{m}\right]$ be a finitely generated commutative graded algebra over $\mathbb{k}$. The Tor-algebra of $P$, denoted by $\operatorname{Tor}_{A}^{*, *}(\mathbb{k}(P), \mathbb{k})$, is a finite-dimensional bigraded $\mathbb{k}-$ algebra. (The explicit definition will be given in Section 2.) Note that the cohomology algebra $H^{*}\left(\mathcal{Z}_{P} ; \mathbb{k}\right)$ of $\mathcal{Z}_{P}$ inherits a canonical bigrading from the Eilenberg-Moore spectral sequence for the fibration

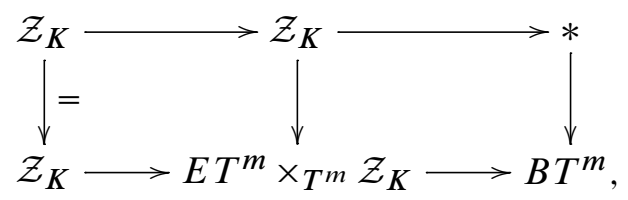

where $E T^{m}$ is a contractible space on which $T^{m}$ acts freely, and $B T^{m}=E T^{m} / T^{m}$. Buchstaber and Panov [2, Theorems 7.6 and 7.7] showed that $H^{*, *}\left(\mathcal{Z}_{P} ; \mathbb{k}\right)$ and $\operatorname{Tor}_{A}^{*, *}(\mathbb{k}(P), \mathbb{k})$ are isomorphic as bigraded $\mathbb{k}$-algebras.

In this study, it is assumed that $\mathbb{k}$ is the field of rational numbers $\mathbb{Q}$. Let $\beta^{-i, 2 j}(P)$ denote the bigraded Betti numbers of the Tor-algebra of $P$ (simply, the bigraded Betti numbers of $P$ ), that is,

$$
\beta^{-i, 2 j}(P)=\operatorname{dim}_{\mathbb{Q}} \operatorname{Tor}_{A}^{-i, 2 j}(\mathbb{Q}(P), \mathbb{Q}) .
$$

Note the bigraded structure should have more information than the usual (mono)graded structure. Hence, it is natural to ask how much information on the topology of $\mathcal{Z}_{P}$ the bigraded Betti numbers contain. Actually, in all known examples (before this paper) of combinatorially different polytopes with the same bigraded Betti numbers (such as vertex truncations of simplices), the moment-angle manifolds are also diffeomorphic. It should also be noted that the bigraded Betti numbers of $H^{*, *}\left(\mathcal{Z}_{P} ; \mathbb{Q}\right)$ are not necessarily topological invariants, although the usual Betti numbers $\beta^{p}=$ $\sum_{p=-i+2 j} \beta^{-i, 2 j}(P)$ are.

From this viewpoint, Panov presented the following problem at the conference on toric topology held in Osaka in November 2011.

Problem 1.2 Let $P$ and $Q$ be two simple polytopes. Is it true that

$$
\mathcal{Z}_{P} \cong \mathcal{Z}_{Q} \quad \Longleftrightarrow \quad \beta^{-i, 2 j}(P)=\beta^{-i, 2 j}(Q) \quad \text { for all } i, j \text { ? }
$$

Here $\cong$ may mean "homotopy equivalent," "homeomorphic" or "diffeomorphic."

In this paper, we answer the "if" part of the problem negatively for all categories: there exist two simple polytopes (say $\mathcal{P}$ and $\mathcal{Q}$ ) with the same bigraded Betti numbers, satisfying $H^{*}\left(\mathcal{Z}_{\mathcal{P}} ; \mathbb{Q}\right) \not H^{*}\left(\mathcal{Z}_{\mathcal{Q}} ; \mathbb{Q}\right)$ as graded rings. Such polytopes are shown in 
Figure 1 and Table 1 presents the complete list of the bigraded Betti numbers of $\mathcal{P}$ and $\mathcal{Q}$.
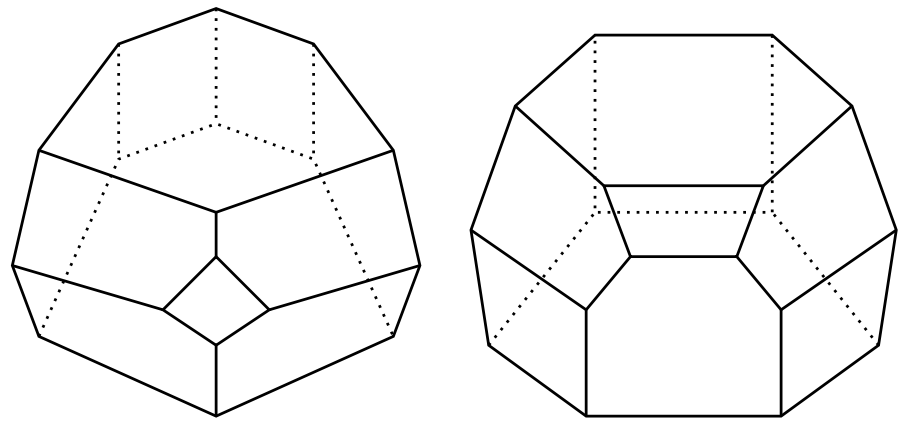

Figure 1: $\mathcal{P}$ and $\mathcal{Q}$

\begin{tabular}{|c|ccccccccc|}
\hline$j \backslash i$ & 0 & 1 & 2 & 3 & 4 & 5 & 6 & 7 & 8 \\
\hline 0 & 1 & & & & & & & & \\
1 & & & & & & & & & \\
2 & & 28 & & & & & & & \\
3 & & 0 & 105 & & & & & & \\
4 & & & 4 & 166 & & & & & \\
5 & & & & 39 & 123 & & & & \\
6 & & & & & 123 & 39 & & & \\
7 & & & & & & 166 & 4 & & \\
8 & & & & & & & 105 & 0 & \\
9 & & & & & & & & 28 & \\
10 & & & & & & & & & \\
11 & & & & & & & & & 1 \\
\hline
\end{tabular}

Table 1: Bigraded Betti numbers of $\mathcal{P}$ and $\mathcal{Q}$

Theorem 1.3 The Tor-algebras of $\mathcal{P}$ and $\mathcal{Q}$ are not isomorphic as bigraded algebras while their bigraded Betti numbers are the same. Furthermore, $H^{*}\left(\mathcal{Z}_{P} ; \mathbb{Q}\right)$ and $H^{*}\left(\mathcal{Z}_{Q} ; \mathbb{Q}\right)$ are not isomorphic as graded rings.

As an immediate corollary, it follows that the bigraded Betti numbers of the simple polytopes do not determine the homotopy type of the corresponding moment-angle manifold.

As by-products, the polytopes $\mathcal{P}$ and $\mathcal{Q}$ are important examples in the toric rigidity problem for simple polytopes as follows. A quasitoric manifold is a closed, smooth 
manifold of dimension $2 n$ that admits a locally standard half-dimensional torus action $T^{n}$ whose orbit space is a simple polytope (see [2] and [7]). A typical example of a quasitoric manifold is a complex projective space $\mathbb{C} P^{n}$ of complex dimension $n$ with the standard $T^{n}$-action, whose orbit space is the $n$-simplex $\Delta^{n}$. Although the topology of a quasitoric manifold does not generally determine the combinatorial type of its orbit space, it sometimes does; for instance, only the $n$-simplex can be the orbit space of a locally standard $T^{n}$-action defined on $\mathbb{C} P^{n}$. Furthermore, since the integral cohomology ring $H^{*}(M ; \mathbb{Z})$ of a quasitoric manifold $M$ can be obtained from the face ring of its orbit polytope $P$, the relationship between the combinatorial type of $P$ and $H^{*}(M ; \mathbb{Z})$ is well established (see the author, Panov and Suh [5] and [7]).

A simple polytope is said to be cohomologically rigid if its combinatorial structure is determined by the cohomology ring of a supporting quasitoric manifold. A simple polytope is said to be combinatorially rigid if its combinatorial structure is determined by the bigraded Betti numbers. By [5, Proposition 3.8], the bigraded Betti numbers of a simple polytope are determined by the cohomology ring of a supporting quasitoric manifold. Hence, any combinatorially rigid polytope (that supports a quasitoric manifold) is cohomologically rigid. However, the question of whether the converse holds has been open (see the author, Masuda and Suh [4, Section 6] for details).

Problem 1.4 [4, Problem 6.6] Find a polytope which is rigid cohomologically but not combinatorially in the set of simple polytopes.

Here, we provide an answer to this problem.

Theorem 1.5 The polytopes $\mathcal{P}$ and $\mathcal{Q}$ are cohomologically rigid, but not combinatorially rigid.

\section{Tor-algebra of a simple polytope}

We briefly review the definitions here, following [2], where the reader may find additional details of the Tor-algebra of a simple polytope, and we present the properties of the multiplicative structure of the Tor-algebra, which are relevant to Section 3.

Let $\mathbb{k}$ be a field, and let $\otimes$ denote $\otimes_{\mathbb{k}}$. Let $A=\mathbb{k}\left[v_{1}, \ldots, v_{m}\right]$ be a finitely generated commutative graded algebra over $\mathbb{k}$. The field $\mathbb{k}$ itself is an $A$-module via the map $A \rightarrow \mathbb{k}$ that sends each $v_{i}$ to 0 . Let $\Lambda\left[u_{1}, \ldots, u_{m}\right]$ denote an exterior algebra on $m$ generators. There is have a differential bigraded algebra $R=\Lambda\left[u_{1}, \ldots, u_{m}\right] \otimes A$ with differential $d: R \rightarrow R$ given by

$$
\operatorname{bideg} u_{i}=(-1,2), \quad \operatorname{bideg} v_{i}=(0,2), \quad d u_{i}=v_{i}, \quad d v_{i}=0 .
$$


Note that $R$ is a free $A$-module. Let $R^{-i}=\Lambda^{i}\left[u_{1}, \ldots, u_{m}\right] \otimes A$, where $\Lambda^{i}\left[u_{1}, \ldots, u_{m}\right]$ is the submodule of $\Lambda\left[u_{1}, \ldots, u_{m}\right]$ spanned by monomials of length $i$. Then, we have the following free resolution of $\mathbb{k}$, which is known as the Koszul resolution:

$$
[R]: 0 \longrightarrow R^{-m} \stackrel{d}{\longrightarrow} \cdots \stackrel{d}{\longrightarrow} R^{-1} \stackrel{d}{\longrightarrow} A \stackrel{d}{\longrightarrow} \mathbb{k} \longrightarrow 0 .
$$

Let $P$ be an $n$-dimensional simple polytope with $m$ facets. The face ring (or the Stanley-Reisner ring) of $P$ is the quotient ring

$$
\mathbb{k}(P)=\mathbb{k}\left[v_{1}, \ldots, v_{m}\right] / I_{P},
$$

where $I_{P}$ is the homogeneous ideal generated by all squarefree monomials $v_{i_{1}} v_{i_{2}} \cdots v_{i_{s}}$ such that $F_{i_{1}} \cap \cdots \cap F_{i_{s}}=\varnothing$. The ideal $I_{P}$ is called the Stanley-Reisner ideal of $P$. By identifying the polynomial ring $\mathbb{k}\left[v_{1}, \ldots, v_{m}\right]$ in the definition of $\mathbb{k}(P)$ with $A$ above, $\mathbb{k}(P)$ can be regarded as an $A$-module. By applying the functor $\otimes_{A} \mathbb{k}(P)$ to the Koszul resolution, we obtain the cochain complex of graded modules

$$
\left[R \otimes_{A} \mathbb{k}(P)\right]: 0 \longrightarrow R^{-m} \otimes_{A} \mathbb{k}(P) \longrightarrow \cdots \longrightarrow R^{-1} \otimes_{A} \mathbb{k}(P) \longrightarrow \mathbb{k}(P),
$$

where the differential map is $d \otimes_{A} 1$. The $(-i)^{\text {th }}$ cohomology module of the above cochain complex is denoted by $\operatorname{Tor}_{A}^{-i}(\mathbb{k}(P), \mathbb{k})$, and we have the graded $A$-module

$$
\operatorname{Tor}_{A}(\mathbb{k}(P), \mathbb{k})=\bigoplus_{i} \operatorname{Tor}_{A}^{-i}(\mathbb{k}(P), \mathbb{k}) .
$$

Note that there is a canonical multiplicative structure on

$$
\operatorname{Tor}_{A}(\mathbb{k}(P), \mathbb{k})=H\left[R \otimes_{A} \mathbb{k}(P)\right]=H\left[\Lambda\left[u_{1}, \ldots, u_{m}\right] \otimes \mathbb{k}(P)\right],
$$

and hence, $\operatorname{Tor}_{A}(\mathbb{k}(P), \mathbb{k})$ is canonically a bigraded $\mathbb{k}$-algebra. The bigraded algebra $\operatorname{Tor}_{A}^{*, *}(\mathbb{k}(P), \mathbb{k})$ is called the Tor-algebra of a simple polytope $P$, and the bigraded Betti numbers of $P$ are defined by

$$
\beta^{-i, 2 j}(P ; \mathbb{k})=\operatorname{dim}_{\mathbb{k}} \operatorname{Tor}_{A}^{-i, 2 j}(\mathbb{k}(P), \mathbb{k}) \quad \text { for } 0 \leq i, j \leq m .
$$

Hereafter, only $\mathbb{k}=\mathbb{Q}$ is considered. For simplicity, we set $\beta^{-i, 2 j}(P)=\beta^{-i, 2 j}(P ; \mathbb{Q})$. The following theorem of Hochster [9] gives a nice combinatorial interpretation of bigraded Betti numbers.

Theorem 2.1 Let $P$ be a simple convex polytope with facets $F_{1}, \ldots, F_{m}$. For a subset $\sigma \subset\{1, \ldots, m\}$, let $P_{\sigma}=\bigcup_{i \in \sigma} F_{i} \subset P$. Then

$$
\beta^{-i, 2 j}(P)=\sum_{|\sigma|=j} \operatorname{dim} \tilde{H}^{j-i-1}\left(P_{\sigma} ; \mathbb{Q}\right) .
$$

Here, $\operatorname{dim} \tilde{H}^{-1}(\varnothing)=1$ by convention. 
Example 2.2 Let $P$ be a 3-dimensional simple polytope with $m$ facets $F_{1}, \ldots, F_{m}$.

(1) $\beta^{-1,4}(P)=\sum_{1 \leq i<j \leq m} \operatorname{dim} \widetilde{H}^{0}\left(F_{i} \cup F_{j} ; \mathbb{Q}\right)$ is the number of pairs of facets that do not intersect.

(2) $\beta^{-1,6}(P)=\sum_{1 \leq i<j<k \leq m} \operatorname{dim} \widetilde{H}^{1}\left(F_{i} \cup F_{j} \cup F_{k}\right.$; $\left.\mathbb{Q}\right)$ is the number of triple of facets whose union is homotopy equivalent to $S^{1}$. Such a triple of facets is called a 3-belt.

(3) A 3-dimensional simple polytope $P$ whose $\beta^{-1,6}(P)$ is 0 is said to be irreducible because it cannot be expressed as a connected sum of a finite number of simple polytopes (see the author and Kim [3]). Assume that $P$ is irreducible. Since there is no 3 -belt, $\beta^{-2,8}(P)$ is equal to the number of quadruples of facets whose union is homotopy equivalent to $S^{1}$. Such a quadruple of facets is called a 4-belt.

Let $P$ be a 3-dimensional simple polytope with $m$ facets $F_{1}, \ldots, F_{m}$. Now, we consider $\Lambda\left[u_{1}, \ldots, u_{m}\right] \otimes \mathbb{k}(P)$. Let $d$ be a differential operator on $\Lambda\left[u_{1}, \ldots, u_{m}\right] \otimes \mathbb{Q}(P)$ induced from $d \otimes_{A} 1$ on $\left[R \otimes_{A} \mathbb{Q}(P)\right]$. As mentioned before, $\operatorname{Tor}_{A}^{*, *}(\mathbb{Q}(P), \mathbb{Q})=$ $H\left[\Lambda\left[u_{1}, \ldots, u_{m}\right] \otimes \mathbb{k}(P)\right]$.

Assume that $F_{i}$ and $F_{j}$ do not intersect. Then, $u_{i} v_{j}$ is an element of bidegree $(-1,4)$ in $\Lambda\left[u_{1}, \ldots, u_{m}\right] \otimes \mathbb{Q}(P)$. Since $d\left(u_{i} v_{j}\right)=v_{i} v_{j}=0 \in \mathbb{Q}(P)$, it is a cycle. Furthermore, $u_{i} v_{j}$ and $v_{i} u_{j}$ are homologous because $d\left(u_{i} u_{j}\right)=v_{i} u_{j}-u_{i} v_{j}$, and $u_{i} v_{j}$ and $u_{i} v_{j^{\prime}}$ are linearly independent in the cohomology group for $\{i, j\} \neq\left\{i^{\prime}, j^{\prime}\right\}$. Therefore, the set of equivalence classes $X^{-1,4}:=\left\{\left[u_{i} v_{j}\right] \mid F_{i} \cap F_{j}=\varnothing\right\}$ becomes a subset of generators of $H^{-1,4}\left[\Lambda\left[u_{1}, \ldots, u_{m}\right] \otimes \mathbb{Q}(P)\right]$. Since $\left|X^{-1,4}\right|=\beta^{-1,4}(P)$, the set $X^{-1,4}$ itself is the set of generators.

Assume that $\beta^{-1,6}(P)=0$ and $\left\{F_{i}, F_{j}, F_{k}, F_{\ell}\right\}$ is a 4-belt of $P$. Then, $u_{i} u_{j} v_{k} v_{\ell}$ is an element of bidegree $(-2,8)$ in $\Lambda\left[u_{1}, \ldots, u_{m}\right] \otimes \mathbb{Q}(P)$. It may be assumed that $F_{i} \cap F_{k}=\varnothing$ and $F_{j} \cap F_{\ell}=\varnothing$. Therefore, $d\left(u_{i} u_{j} v_{k} v_{\ell}\right)=0$. One can easily see that $u_{i} u_{j} v_{k} v_{\ell}$ is homologous to $u_{i^{\prime}} u_{j^{\prime}} v_{k^{\prime}} v_{\ell^{\prime}}$ if and only if the 4-tuple $(i, j, k, \ell)$ is obtained from $\left(i^{\prime}, j^{\prime}, k^{\prime}, \ell^{\prime}\right)$ by a cyclical shift and, perhaps, a reflection. Let $X^{-2,8}:=\left\{\left[u_{i} u_{j} v_{k} v_{\ell}\right] \mid\left\{F_{i}, F_{j}, F_{k}, F_{\ell}\right\}\right.$ is a 4 -belt $\}$, which is a subset of generators of $H^{-2,8}\left[\Lambda\left[u_{1}, \ldots, u_{m}\right] \otimes \mathbb{Q}(P)\right]$. Since $\left|X^{-2,8}\right|=\beta^{-2,8}(P)$, the set $X^{-1,4}$ itself is the set of generators. Hence, we have the following proposition.

Proposition 2.3 If $P$ is a 3-dimensional simple polytope with $m$ facets $F_{1}, \ldots, F_{m}$, with $\beta^{-1,6}(P)=0$, then each generator of $\operatorname{Tor}_{A}^{-1,4}(\mathbb{Q}(P), \mathbb{Q})$ can be indexed by a pair of facets that do not intersect, and each generator of $\operatorname{Tor}_{A}^{-2,8}(\mathbb{Q}(P), \mathbb{Q})$ can 
be indexed by $4-$ belts. Let $\sigma$ and $\tau$ be generators in $\operatorname{Tor}_{A}^{-1,4}(\mathbb{Q}(P), \mathbb{Q})$ indexed by $\left\{F_{1}, F_{3}\right\}$ and $\left\{F_{2}, F_{4}\right\}$, respectively. Then,

$$
\sigma \cdot \tau= \begin{cases}\eta \neq 0 & \text { if }\left\{F_{1}, F_{2}, F_{3}, F_{4}\right\} \text { is a 4-belt in } P, \\ 0 & \text { otherwise, }\end{cases}
$$

where $\eta$ is a generator in $\operatorname{Tor}_{A}^{-2,8}(\mathbb{Q}(P), \mathbb{Q})$ indexed by $\left\{F_{1}, F_{2}, F_{3}, F_{4}\right\}$.

\section{Proof of Theorem 1.3}

Note that $\operatorname{Tor}_{A}^{*, *}(\mathbb{Q}(\mathcal{P}), \mathbb{Q})$ and $\operatorname{Tor}_{A}^{*, *}(\mathbb{Q}(\mathcal{Q}), \mathbb{Q})$ are isomorphic as groups. Hence, their multiplicative structures should be compared. We index each facet of $\mathcal{P}$ and $\mathcal{Q}$ as shown in Figure 2.
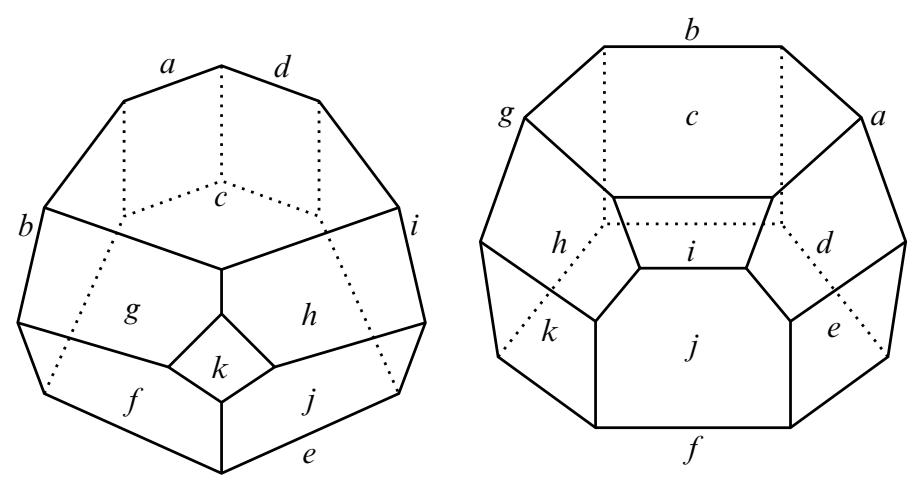

Figure 2: Indices of facets of $\mathcal{P}$ and $\mathcal{Q}$

Let $A=\mathbb{Q}[a, b, \ldots, k]$, where the letters are degree 2 indeterminates corresponding to the facets of $\mathcal{P}$. The bigraded Betti numbers of $\mathcal{P}$ and $\mathcal{Q}$ can be computed by the algebra program Macaulay2 [8] as in Figure 3. Then, one can see that they have the same bigraded Betti numbers.

Now, consider the subspace

$$
V_{P}:=\left\{x \in \operatorname{Tor}_{A}^{-1,4}(\mathbb{Q}(P), \mathbb{Q}) \mid x r=0 \text { for all } r \in \operatorname{Tor}_{A}^{-1,4}(\mathbb{Q}(P), \mathbb{Q})\right\}
$$

as a vector space over $\mathbb{Q}$. It is obvious that the dimension of $V_{P}$ is a ring invariant of $\operatorname{Tor}_{A}(\mathbb{Q}(P), \mathbb{Q})$.

Note that $\beta^{-2,8}(\mathcal{P})=\beta^{-2,8}(\mathcal{Q})=4$. In other words, there are four 4 -belts in both $\mathcal{P}$ and $\mathcal{Q}$. In $\mathcal{P}$, all 4 -belts are indexed by $\{b, c, d, e\},\{g, f, j, h\},\{a, c, i, e\}$ and 
Macaulay2, version 1.4

with packages: ConwayPolynomials, Elimination, IntegralClosure, LLLBases, PrimaryDecomposition, ReesAlgebra, TangentCone

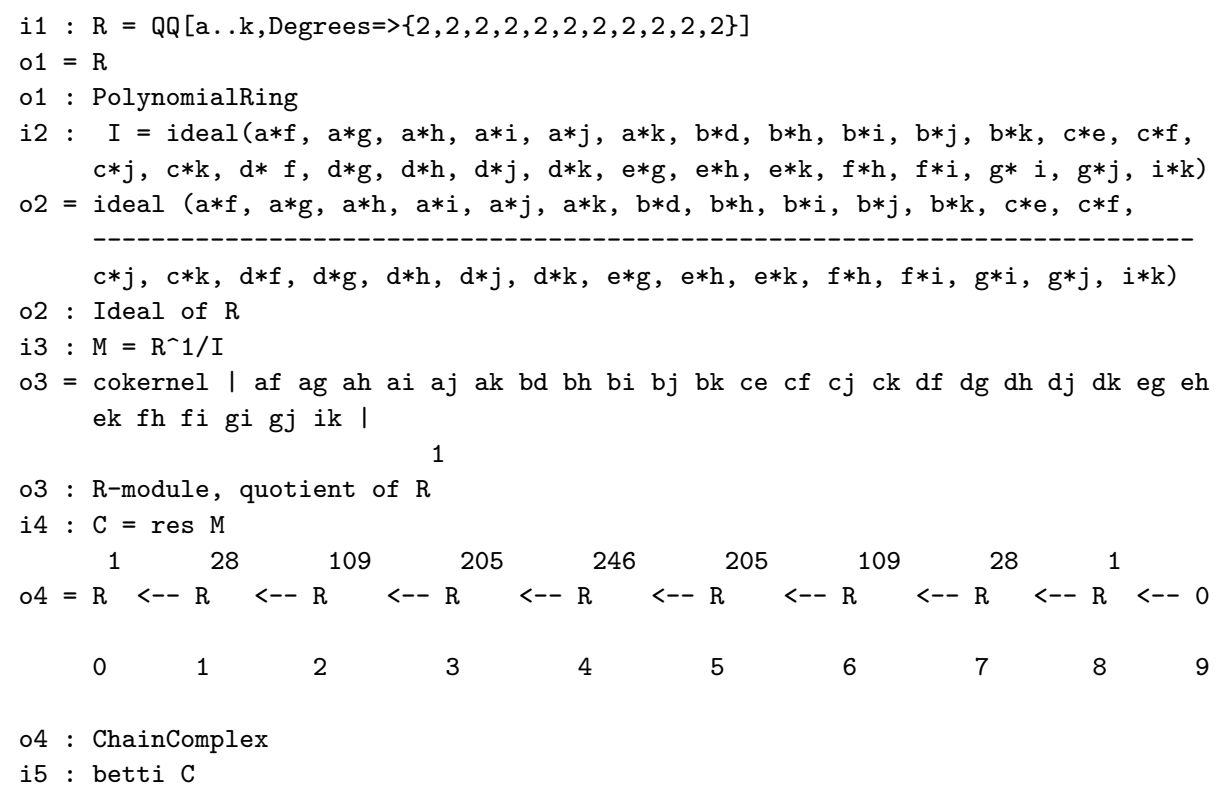

o5 : BettiTally

Figure 3: Program code for computing $\beta^{*, *}(\mathcal{P})$

$\{c, i, e, b\}$. Hence, only some of the products between two pairs among $\{b, d\},\{c, e\}$, $\{g, j\},\{f, h\},\{a, i\}$ and $\{b, i\}$ (6 generators) can be nonzero. This implies that $\operatorname{dim}_{\mathbb{Q}} V_{P}=28-6=22$. 
In $\mathcal{Q}$, all 4-belts are indexed by $\{a, c, g, f\},\{a, d, j, f\},\{c, h, j, d\}$ and $\{f, g, h, j\}$. Hence, only some of the products between two pairs among $\{a, g\},\{c, f\},\{a, j\}$, $\{d, f\},\{c, j\},\{h, d\},\{f, h\}$ and $\{g, j\}$ (8 generators) can be nonzero. This implies that $\operatorname{dim}_{\mathbb{Q}} V_{Q}=28-8=20$. Therefore, $\operatorname{Tor}_{A}(\mathbb{Q}(\mathcal{P}), \mathbb{Q})$ and $\operatorname{Tor}_{A}(\mathbb{Q}(\mathcal{Q}), \mathbb{Q})$ are not isomorphic as bigraded algebras, which proves the first part of the theorem.

Furthermore, since $H^{3}\left(\mathcal{Z}_{P} ; \mathbb{Q}\right)=\operatorname{Tor}_{A}^{-1,4}(\mathbb{Q}(P), \mathbb{Q})$ one can show $H^{*}\left(\mathcal{Z}_{\mathcal{P}} ; \mathbb{Q}\right) \not$ $H^{*}\left(\mathcal{Z}_{\mathcal{Q}} ; \mathbb{Q}\right)$ as graded rings by using the above argument, which proves the second part of the theorem.

\section{Proof of Theorem 1.5}

First, it is obvious that $\mathcal{P}$ and $\mathcal{Q}$ are not combinatorially rigid, since they have the same bigraded Betti numbers but are not combinatorially equivalent.

In the remaining part of this section, we prove that $\mathcal{P}$ and $\mathcal{Q}$ are cohomologically rigid.

Suppose that there exists a polytope $P^{\prime}$ and quasitoric manifolds $M$ and $N$ over $\mathcal{P}$ and $P^{\prime}$, respectively, such that $H^{*}(M ; \mathbb{Z})$ and $H^{*}(N ; \mathbb{Z})$ are isomorphic as graded rings. Then, by [5, Lemma 3.7], it follows that $P^{\prime}$ has 11 facets, and

$$
\operatorname{Tor}_{A}^{*, *}(\mathbb{Q}(\mathcal{P}), \mathbb{Q})=\operatorname{Tor}_{A}^{*, *}\left(\mathbb{Q}\left(P^{\prime}\right), \mathbb{Q}\right) .
$$

In particular, $\beta^{*, *}(\mathcal{P})=\beta^{*, *}\left(P^{\prime}\right)$.

Now, let us investigate all other polytopes with 11 facets. A graph $G$ is said to be $P^{3}$-realizable if there is a 3 -dimensional polytope whose corresponding 1-complex is isomorphic to $G$. Let $P(G)$ denote such a polytope. A graph $G$ is said to be $k-$ (vertex-)connected if there is no set of $k-1$ vertices that, when removed, disconnects the graph. It is known that a graph $G$ is planar and 3 -connected if and only if $G$ is $P^{3}$-realizable (Steinitz [10]). A $P^{3}$-realizable graph is called a triangulation if all the faces of the graph are triangles when the graph is embedded into a 2-dimensional sphere $S^{2}$. Hence if $G$ is a 3 -connected triangulation, then $P(G)$ is a simplicial polytope that is dual to a simple polytope.

Note that both $\mathcal{P}$ and $\mathcal{Q}$ are 3-dimensional simple polytopes having 11 facets. Using the graph-generating program plantri [1], developed by Brinkmann and McKay, we can list all 3-connected triangulations with a certain number of vertices. Such a list gives us all 3-dimensional simple polytopes with a certain number of facets. Using the program Macaulay2 again, we can list all 3-dimensional simple polytopes $P$ with 11 facets satisfying $\beta^{-1,6}(P)=0$ and compute their bigraded Betti numbers (see the author [6]). See Table 2; each polytope has 11 facets $a, b, \ldots, k$. Each polytope is 


\begin{tabular}{|c|c|c|}
\hline & Polytope & Betti number \\
\hline 1 & bcdef,afghc,abhid,acijke,adkf,aekgb,bfkjh,bgjic,chjd,dihgk,djgfe & $(28,105,164,112,28,2,0)$ \\
\hline 2 & bcdef,afghijc,abjd,acjke,adkgf,aegb,bfekh,bgki,bhkj,bikdc,djihge & $(28,105,167,131,47,5,0)$ \\
\hline 3 & bcde,aefghic,abid,acijke,adkfb,bekjg,bfjh,bgji,bhjdc,dihgfk,djfe & $(28,105,169,138,54,7,0)$ \\
\hline 4 & bcde,aefghijc,abjd,acjke,adkfb,bekg,bfkh,bgki,bhkj,bikdc,djihgfe & $(28,105,175,159,75,13,0)$ \\
\hline 5 & bcdef,afghijc,abjd,acje,adjkf,aekgb,bfkh,bgki,bhkj,bikedc,ejihgf & $(28,105,172,144,60,10,0)$ \\
\hline 6 & bcde,aefc,abfgd,acghe,adhijfb,bejgc,cfjkhd,dgkie,ehkj,eikgf,gjih & $(28,105,171,141,57,9,0)$ \\
\hline 7 & bcde,aefgc,abghijkd,acke,adkjfb,bejihg,bfhc,cgfi,chfj,cifek,cjed & $(28,105,174,156,72,12,0)$ \\
\hline 8 & bcde,aefghc,abhijd,acje,adjfb,bejkg,bfkh,bgkic,chkj,cikfed,fjihg & $(28,105,168,129,45,6,0)$ \\
\hline 9 & bcde,aefghc,abhijd,acje,adjfb,bejikg,bfkh,bgkic,chkfj,cifed,fihg & $(28,105,170,136,52,8,0)$ \\
\hline 10 & bcdef,afghic,abid,acijke,adkf,aekgb,bfkjh,bgji,bhjdc,dihgk,djgfe & $(28,105,165,119,35,3,0)$ \\
\hline 11 & bcde,aefghic,abid,acije,adjkfb,bekg,bfkh,bgkji,bhjdc,dihke,ejhgf & $(28,105,170,136,52,8,0)$ \\
\hline 12 & bcde,aefgc,abghid,acie,adijfb,bejkg,bfkhc,cgkji,chjed,eihkf,fjhg & $(28,105,166,123,39,4,0)$ \\
\hline 13 & bcde,aefgc,abghid,acie,adijfb,bejkg,bfkhc,cgki,chkjed,eikf,fjihg & $(28,105,167,125,41,5,0)$ \\
\hline 14 & bcde,aefghc,abhd,achije,adjfb,bejikg,bfkh,bgkidc,dhkfj,dife,fihg & $(28,105,169,134,50,7,0)$ \\
\hline 15 & bcde,aefghc,abhijd,acjgfe,adfb,bedg,bfdjkh,bgkic,chkj,cikgd,gjih & $(28,105,173,145,61,11,0)$ \\
\hline 16 & bcde,aefc,abfghid,acie,adijkfb,bekgc,cfkjh,cgji,chjed,eihgk,ejgf & $(28,105,170,143,59,8,0)$ \\
\hline 17 & bcde,aefc,abfghid,acie,adihjfb,bejkgc,cfkh,cgkjei,ched,ehkf,fjhg & $(28,105,177,159,75,15,0)$ \\
\hline 18 & bcde,aefghic,abid,acijgke,adkfb,bekg,bfkdjh,bgji,bhjdc,dihg,dgfe & $(28,105,173,149,65,11,0)$ \\
\hline 19 & bcde,aefghijc,abjd,acjkhgfe,adfb,bedg,bfdh,bgdki,bhkj,bikdc,djih & $(28,105,179,169,85,17,0)$ \\
\hline 20 & bcde,aefghijkc,abkd,ackjihgfe,adfb,bedg,bfdh,bgdi,bhdj,bidk,bjdc & $(28,105,189,189,105,27,0)$ \\
\hline 21 & bcde,aefgc,abghd,ache,adhijfb,bejg,bfjkhc,cgkied,ehkj,eikgf,gjih & $(28,105,171,141,57,9,0)$ \\
\hline 22 & bcde,aefc,abfghd,ache,adhijfb,bejkgc,cfkh,cgkied,ehkj,eikf,fjihg & $(28,105,173,145,61,11,0)$ \\
\hline 23 & bcdefg,aghc,abhijd,acje,adjf,aejkhg,afhb,bgfkic,chkj,cikfed,fjih & $(28,105,171,137,53,9,0)$ \\
\hline 24 & bcdef,afgc,abghid,acije,adjf,aejkgb,bfkhc,cgkji,chjd,dihkfe,fjhg & $(28,105,166,123,39,4,0)$ \\
\hline 25 & bcdef,afgc,abghijd,acje,adjkhgf,aegb,bfehc,cgeki,chkj,ciked,ejih & $(28,105,173,149,65,11,0)$ \\
\hline
\end{tabular}

Table 2: Irreducible polytopes with 11 facets

indexed by adjacency of the facets. The $n^{\text {th }}$ component is the list of facets that intersect the (alphabetical) $n^{\text {th }}$ facet. The Betti numbers are listed in the form

$$
\left(\beta^{-1,4}(P), \ldots, \beta^{-(j-1), 2 j}(P), \ldots, \beta^{-7,16}(P)\right)
$$

Note that the integer tuple of the form above completely determines all the bigraded Betti numbers of a 3-dimensional polytope (see [5, Section 7] for details).

In Table 2 , the $12^{\text {th }}$ polytope is $\mathcal{P}$, and the $24^{\text {th }}$ polytope is $\mathcal{Q}$. One can easily check that there is no other polytope whose bigraded Betti numbers are equal to those of $\mathcal{P}$ and $\mathcal{Q}$. Thus, $P^{\prime}$ cannot be combinatorially equivalent to any polytope with 11 facets other than $\mathcal{P}$ and $\mathcal{Q}$. Moreover, by Theorem $1.3, P^{\prime}$ cannot be combinatorially equivalent to $\mathcal{Q}$. Therefore, $P^{\prime}$ is $\mathcal{P}$, which proves that $\mathcal{P}$ is cohomologically rigid.

Similar arguments can be presented for $\mathcal{Q}$ to prove its cohomological rigidity. 
Acknowledgements The author was supported by Basic Science Research Program through the National Research Foundation of Korea (NRF) funded by the Ministry of Education (NRF-2011-0024975) and the TJ Park Science Fellowship funded by the POSCO TJ Park Foundation.

\section{References}

[1] G Brinkmann, B McKay, plantri (2011) Available at http://cs.anu.edu.au/ $\sim$ bdm/plantri/

[2] V M Buchstaber, T E Panov, Torus actions and their applications in topology and combinatorics, University Lecture Series 24, Amer. Math. Soc. (2002) MR1897064

[3] S Choi, J S Kim, Combinatorial rigidity of 3-dimensional simplicial polytopes, Int. Math. Res. Not. 2011 (2011) 1935-1951 MR2806527

[4] S Choi, M Masuda, D Y Suh, Rigidity problems in toric topology: A survey, Tr. Mat. Inst. Steklova 275 (2011) 188-201 MR2962979

[5] S Choi, T Panov, D Y Suh, Toric cohomological rigidity of simple convex polytopes, J. Lond. Math. Soc. 82 (2010) 343-360 MR2725043

[6] Y Choi, Cohomological rigidity of simple 3-polytopes with 10 facets, master's thesis, Korea Advanced Institute of Science and Technology (2008)

[7] M W Davis, T Januszkiewicz, Convex polytopes, Coxeter orbifolds and torus actions, Duke Math. J. 62 (1991) 417-451 MR1104531

[8] D Eisenbud, D Grayson, M Stillman, Macaulay2 (2013) Available at http:// www.math.uiuc.edu/Macaulay2/

[9] M Hochster, Cohen-Macaulay rings, combinatorics, and simplicial complexes, from: "Ring theory, II (Proceedings of the Second Oklahoma Ring Theory Conference)", (B R McDonald, R A Morris, editors), Lecture Notes in Pure and Appl. Math. 26, Dekker, New York (1977) 171-223 MR0441987

[10] E Steinitz, Polyeder und Raumeinteilungen, from: "Enzykl. Math. Wiss.", (F Klein, W Meyer, editors), volume 3, B G Teubner Verlag, Leipzig (1922) 1-139

Department of Mathematics, Ajou University

San 5, Woncheon-dong, Yeongtong-gu, Suwon 443-749, South Korea schoi@ajou.ac.kr

Received: 31 July 2012 Revised: 4 June 2013 